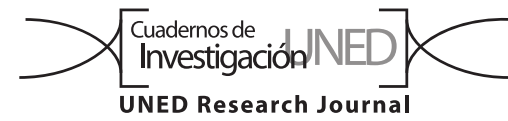

\title{
Nivel de competencias del personal docente a distancia en la cátedra de Agroindustria en una universidad a distancia
}

\author{
Carmen Andrés Jiménez ${ }^{1} \&$ Jacqueline Alfaro Azofeifa ${ }^{2}$ \\ 1. Universidad Estatal a Distancia (UNED), 474-2050 Mercedes de Montes de Oca, San José-Costa Rica; candres@uned.ac.cr \\ 2. Universidad Estatal a Distancia (UNED), 474-2050 Mercedes de Montes de Oca, San José-Costa Rica; jalfaro@uned.ac.cr
}

Recibido 01-XI-2017 • Corregido 07-XII-2017 • Aceptado 16-I-2018

\begin{abstract}
Competency level of teachers in the Agroindustry chair in a distance university. In distance education, tutors need skills such as accessibility and motivation; virtual socialization; information exchange, and knowledge construction and development. We determined which of these skills were being applied in two courses of a Costa Rican distance university. We used a validated survey applied to all the teachers of the Agroindustry chair (three) and validated checklist applied to two courses. We only found satisfactory results in one course. Tutors have a tendency towards planning; stimulating collaborative work among students; criteria unification and use of pedagogical strategies. On the other hand, we realized how some tutors do not identify the way students learn; knowledge networks are not created as well as mutual knowledge outside the classroom. Finally, some tutors do not have formal training in virtual classrooms, although this did not have a negative impact in their performance as instructors. We recommend the implementation of formal training, collaborative workspaces and deeper acquaintance with the students.
\end{abstract}

Key words: distance tutor, online tutor, distance education, educational competences.
RESUMEN: En la educación a distancia, los tutores necesitan una serie de habilidades como la accesibilidad y la motivación, socialización virtual, intercambio de información y construcción del conocimiento, así como el desarrollo. Determinamos cuáles de estas habilidades se estaban aplicando en dos asignaturas de una universidad a distancia en Costa Rica. Utilizamos una encuesta validada aplicada a las tutoras de la cátedra de Agroindustria (tres) y una lista de chequeo también validada, aplicada a dos asignaturas de la cátedra. Encontramos resultados satisfactorios en una de las asignaturas evaluadas. Las tutoras tienen la tendencia a planificar sus lecciones, estimular el trabajo colaborativo entre el estudiantado, unificación de criterio y el uso de estrategias pedagógicas. Sin embargo, encontramos que algunas tutoras no identifican la manera en la que los estudiantes aprenden, no generan redes de conocimiento para poder extenderlo fuera de las aulas virtuales. Finalmente, algunas tutoras no tienen un entrenamiento formal en clases virtuales, aunque eso no tuvo un impacto negativo en su rendimiento como instructoras. Recomendamos la implementación de un entrenamiento formal, espacios de trabajo colaborativo y fomentar un conocimiento más profundo de la comunidad estudiantil.

Palabras clave: tutoría a distancia, tutoría en línea, educación a distancia, competencias educativas.
Para cumplir los objetivos de aprendizaje y ofertar una asignatura de calidad, se deben abarcar tres ejes fundamentales a saber: un buen uso de las herramientas tecnológicas por parte los actores en el proceso de enseñanza-aprendizaje, desarrollo de competencias en el personal docente a distancia y una aplicación correcta de la mediación pedagógica que en consecuencia alcanzará la autorregulación y la metacognición en los estudiantes. Con respecto al primer eje, la evolución de las nuevas tecnologías ha propiciado un aprendizaje diferenciado como lo es la educación virtual (Cabero \& Román, 2008). De acuerdo al modelo pedagógico de la UNED, es preciso encontrar metodologías y estrategias de educación a distancia apropiadas que permitan aprovechar todo el potencial de las tecnologías al servicio de una formación más autónoma de todos aquellos que deseen hacerlo (UNED, 2004).

A esto se puede agregar que, si bien el estudiantado y el profesorado están separados físicamente, los entornos virtuales de aprendizaje tienen el fin básico de favorecer la interacción. Esta es una de las funciones primordiales en el cual el personal docente debe de propiciar estos entornos (Calvo \& Salas, s.f).

Ahora bien, de acuerdo con García (2014), el personal docente a distancia, en su mayoría, ha sido formado 
con métodos convencionales de enseñanza y el hecho de que la convergencia entre los modelos presenciales y a distancia cada vez sea más patente, nos obliga a pasar del aprendizaje dirigido basado en memorización y reproducción a uno más autónomo, reflexivo, racional y aplicativo de conocimientos. De esto se trata el segundo eje para ofrecer una asignatura de calidad.

Entonces, por ejemplo, Salas (s.f) indican que ser profesor en educación a distancia implica una integración de propuestas de e-learning para lo cual debe estar preparado y ser capaz de discernir cuales de estas posibilidades le brindan una mejor opción para garantizar una enseñanza de calidad. Estas ideas se hacen evidentes con lo que indica García (2014), referente al compromiso que se espera del personal docente para mejorar la calidad de las asignaturas que imparte, para lo cual debe enfocarse en el ámbito laboral-profesional, ético, formativo, de actualización, colaborativo, disciplinar y pedagógico/ didáctico, tecnológico, investigador e innovador, los cuales interactúan entre sí para lograr un trabajo de calidad.

Desde otra posición, Salmon en el 2004, citado por Salas (s.f) indica los cinco niveles de intervención dentro de la función de moderador y facilitador de un tutor, entre los cuales se encuentran: acceso y motivación, la socialización en línea, manejo e intercambio de información, construcción del conocimiento y desarrollo; en el cual el personal docente debe propiciar el desarrollo cognitivo del estudiantado.

Otro aspecto en lo que el personal docente debe involucrarse es el evaluar para aprender, el cual, es un tema sobre el cual las tutoras involucradas en la cátedra de Agroindustria han estado aprendiendo. Al respecto, García, en el año 2013, mencionado por Jiménez (2016), indicó que el objeto de evaluar para aprender es que el educando, paulatinamente, desarrolle habilidades (cognitivas y emocionales) para "aprender a aprender; es decir, que se comprometa en construir su conocimiento, a partir de aprendizajes previos, con el fin de valerse por sí mismo para utilizar y aplicar el conocimiento en diferentes contextos".

Finalmente, el tercer eje que garantiza una educación de calidad involucra a la población estudiantil. Como lo indican Castillo, Torres y González (2009), el estudiantado a distancia posee competencias diferenciadas con respecto al presencial, dado que estos desarrollan más iniciativas al ser la enseñanza más flexible y abierta. Pero para alcanzar este objetivo, es necesario un trabajo colaborativo que involucra tanto al personal docente como al estudiantado.

La hipótesis de esta sistematización de experiencia es que si se logra que el personal docente a distancia de la cátedra de Agroindustria trabaje a la luz de las competencias que debe tener, según lo que la teoría establece, junto con un buen uso de las herramientas tecnológicas y la mediación pedagógica; se podrá ofrecer asignaturas de calidad que cumplan con las expectativas del estudiantado.

El objetivo de esta experiencia fue comparar las competencias que el personal docente de la cátedra de Agroindustria aplica en las asignaturas que imparte con las que en teoría debe tener y generar a partir de ahí un plan de mejora.

\section{METODOLOGÍA}

Se realizó un estudio descriptivo que conforma una sistematización de una experiencia para valorar el nivel de cumplimiento del personal docente de una cátedra, a la luz de las competencias que en la teoría debe de tener. A continuación, se presenta la secuencia de acciones que llevaron a este resultado:

Investigación previa sobre competencias que debe tener un tutor a distancia: Se realizó una investigación teórica sobre las competencias que el personal docente debe tener de acuerdo a varios autores y a partir de ella se elaboró la lista de chequeo que está en el apéndice 1.

En esta lista se incluyeron temas desde el uso de la plataforma y los recursos innovadores como medio de aprendizaje, la organización previa como docente hasta las estrategias utilizadas para motivar al estudiante a participar activamente, ser crítico y en su proceso de aprendizaje y lograr una correcta evaluación de los aprendizajes.

Asimismo, esta investigación se muestra en la introducción del documento y en la sección de resultados se muestra un cuadro con las competencias más relevantes encontradas a nivel práctico.

\section{Encuesta al personal docente de la Cátedra de} Agroindustria: A partir de la investigación realizada en el punto anterior, se diseñó una encuesta validada por dos expertos en educación a distancia y elaboración de encuestas (Apéndice 2). De esta manera, se logró plasmar en las preguntas a realizar la información correcta que determinaría el nivel de cumplimiento del personal y así lograr que su trabajo como mediadores en un proceso a distancia sea el adecuado. Los temas más importantes que se trataron fueron: planificación previa, atención y motivación adecuada al estudiantado, mediación de 
los contenidos y fomento de la criticidad, uso adecuado de la tecnología y evaluación de los aprendizajes.

Por otro lado, la encuesta incluía un consentimiento informado en el que se le informaba al tutor de la cátedra de Agroindustria que la información que entregara sería tratada de manera confidencial y no tendrá injerencia alguna sobre su evaluación del desempeño o cualquier otro rubro que pudiera perjudicarle en su desempeño como tutor de la UNED.

Selección de muestra: Una vez que se preparó el instrumento con el cual se iba a recopilar la información, se seleccionó una muestra de dos asignaturas impartidas por la cátedra de Agroindustria durante el segundo cuatrimestre del 2017, las cuales eran: Calidad Agroalimentaria y Gerencia de Empresas Agropecuarias y Agroindustriales. Esta muestra de asignaturas se escogió por tener tanto componentes en línea como prácticos, además de que tienen características muy específicas y son muy diferentes una a la otra, por lo que los resultados que se obtuvieran enriquecerían la investigación.

Análisis de asignaturas para comprobar la aplicación de las competencias necesarias: Se elaboró una lista de chequeo a partir de la investigación realizada en el punto uno, en donde se analizaron las asignaturas con que cuenta la cátedra, para así determinar cuánto de lo requerido está siendo implementado por parte del personal docente.

Un aspecto importante es que la cátedra de Agroindustria está compuesta por tres tutoras y la encargada, quien tiene la función de diseñar los entornos virtuales y las actividades evaluativas, así como los instrumentos de evaluación; razón por la cual para aplicar esta lista de chequeo, se solicitó a personas expertas externas que revisaran el entorno y todos los recursos, así como actividades e instrumentos de evaluación, para tomar nota de las sugerencias y errores que se puedan estar cometiendo. De esta manera, a través de los resultados que se obtuvieran, se elaboraría un plan de acción que pudiera mejorar la calidad a las asignaturas.

\section{RESULTADOS}

Encuesta al personal docente de la cátedra de Agroindustria: No todas las tutoras identifican la manera en la que cada estudiante aprende, ni crean comunidades de aprendizaje. Algunas no han recibido el curso: "Diseño de cursos en línea"y no todos se preocupan por el cumplimiento de los objetivos de aprendizaje (Cuadro 1).

CUADRO 1

Resultados de la encuesta sobre las competencias del personal docente de la cátedra de Agroindustria.

\begin{tabular}{|c|c|c|c|c|c|c|c|c|c|}
\hline Criterio & \multicolumn{9}{|c|}{ Características } \\
\hline \multirow[t]{3}{*}{$\begin{array}{l}\text { Planificación de sus estrategias } \\
\text { didácticas, apropiadas al estudiante }\end{array}$} & \multicolumn{3}{|c|}{$\begin{array}{l}\text { Identificar la manera en la } \\
\text { que cada estudiante aprende }\end{array}$} & \multicolumn{3}{|c|}{$\begin{array}{l}\text { Se llevan a cabo procesos de } \\
\text { interacción, reflexión y análisis }\end{array}$} & \multicolumn{3}{|c|}{$\begin{array}{c}\text { Se monitorea la participación } \\
\text { de todos el estudiantado }\end{array}$} \\
\hline & $\mathrm{T} 1^{*}$ & $\mathrm{~T} 2$ & T3 & T1 & $\mathrm{T} 2$ & T3 & $\mathrm{T} 1$ & $\mathrm{~T} 2$ & T3 \\
\hline & Sí & No & No & Sí & Sí & Sí & Sí & Sí & Sí \\
\hline
\end{tabular}

\begin{tabular}{|c|c|c|c|c|c|c|c|c|c|c|c|c|}
\hline \multirow{3}{*}{$\begin{array}{l}\text { Criterio } \\
\text { Características que se } \\
\text { emplean en las asignaturas }\end{array}$} & \multicolumn{12}{|c|}{ Características } \\
\hline & \multicolumn{3}{|c|}{$\begin{array}{l}\text { Creación de comunidades } \\
\text { de aprendizaje }\end{array}$} & \multicolumn{3}{|c|}{$\begin{array}{l}\text { Estimulación de la } \\
\text { construcción conjunta } \\
\text { de conocimiento }\end{array}$} & \multicolumn{3}{|c|}{$\begin{array}{c}\text { Áreas de consenso y } \\
\text { diversidad de criterios }\end{array}$} & \multicolumn{3}{|c|}{$\begin{array}{c}\text { Espacios de conocimiento } \\
\text { mutuo fuera del área } \\
\text { de conocimiento }\end{array}$} \\
\hline & T1 & $\mathrm{T} 2$ & T3 & T1 & $\mathrm{T} 2$ & T3 & T1 & $\mathrm{T} 2$ & T3 & T1 & $\mathrm{T} 2$ & T3 \\
\hline & Sí & No & No & Sí & Sí & Sí & Sí & Sí & No & No & Sí & No \\
\hline
\end{tabular}

\begin{tabular}{|c|c|c|c|c|c|c|c|c|c|c|c|c|c|c|c|c|c|c|}
\hline Criterio & \multicolumn{18}{|c|}{ Características } \\
\hline \multirow[t]{3}{*}{$\begin{array}{l}\text { Estrategias } \\
\text { pedagógicas }\end{array}$} & \multicolumn{3}{|c|}{$\begin{array}{c}\text { Atención de } \\
\text { dudas }\end{array}$} & \multicolumn{3}{|c|}{$\begin{array}{c}\text { Estimular destrezas } \\
\text { metacognitivas }\end{array}$} & \multicolumn{3}{|c|}{$\begin{array}{l}\text { Ser guía en } \\
\text { procesos de } \\
\text { aprendizaje }\end{array}$} & \multicolumn{3}{|c|}{$\begin{array}{c}\text { Desarrollo de } \\
\text { materiales para } \\
\text { necesidades especiales }\end{array}$} & \multicolumn{3}{|c|}{$\begin{array}{l}\text { Pensamiento } \\
\text { reflexivo y crítico }\end{array}$} & \multicolumn{3}{|c|}{$\begin{array}{l}\text { Cumplimiento de } \\
\text { los objetivos } \\
\text { del curso }\end{array}$} \\
\hline & T1 & $\mathrm{T} 2$ & T3 & T1 & $\mathrm{T} 2$ & T3 & $\mathrm{T} 1$ & $\mathrm{~T} 2$ & T3 & $\mathrm{T} 1$ & $\mathrm{~T} 2$ & T3 & $\mathrm{T} 1$ & $\mathrm{~T} 2$ & T3 & $\mathrm{T} 1$ & $\mathrm{~T} 2$ & T3 \\
\hline & Sí & Sí & Sí & Sí & Sí & Sí & Sí & Sí & Sí & Sí & Sí & Sí & Sí & Sí & Sí & Sí & No & Sí \\
\hline
\end{tabular}


CUADRO 2 (Continuación)

\begin{tabular}{|c|c|c|c|}
\hline \multirow{3}{*}{\begin{tabular}{l}
\multicolumn{1}{c}{ Criterio } \\
Conocimiento del \\
plan de estudios y \\
diseño curricular
\end{tabular}} & \multicolumn{3}{|c|}{ Características } \\
\hline & $\mathrm{T} 1$ & $\mathrm{~T} 2$ & T3 \\
\hline & $\begin{array}{l}\text { Manifiesta tener conocimiento } \\
\text { del plan de estudios y del diseño } \\
\text { curricular de las asignaturas. }\end{array}$ & $\begin{array}{l}\text { Manifiesta tener conocimiento } \\
\text { del plan de estudios y del diseño } \\
\text { curricular de las asignaturas. }\end{array}$ & $\begin{array}{l}\text { No manifiesta tener conocimiento } \\
\text { del plan de estudios y del diseño } \\
\text { curricular de las asignaturas. }\end{array}$ \\
\hline
\end{tabular}

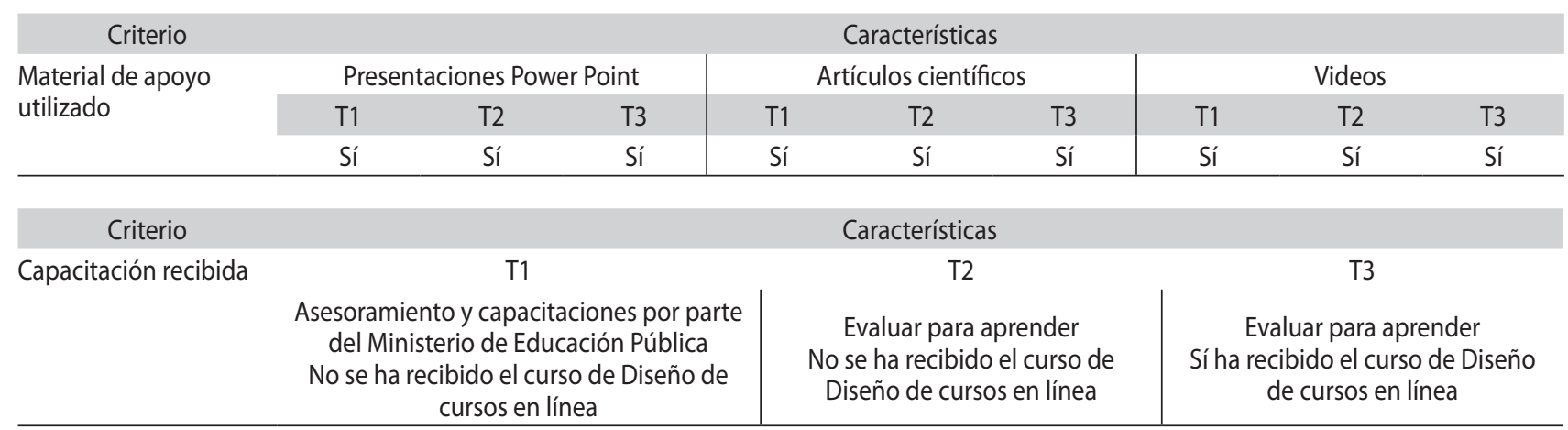

*Tutora 1, 2 y 3.

Comparación entre las asignaturas para comprobar la aplicación de las competencias necesarias: Se observa que la planificación de estrategias didácticas basadas en las necesidades del estudiantado y la creación de comunidades de aprendizaje, así como la apertura de espacios para la socialización entre estudiantes y el personal docente, se observa que no están presentes en los entornos virtuales (Cuadro 2).

Se encontró que hay una diferencia entre las dos asignaturas estudiadas, dado que en Calidad Agroalimentaria hay mayor mediación y mayor fomento de la criticidad además de participación del estudiantado que en Gerencia de Empresas Agropecuarias y Agroindustriales (Cuadro 3).

De forma general, se puede ver que las tutoras están utilizando las herramientas tecnológicas de la mejor manera, a excepción de que falta la incorporación de nuevas tecnologías y recursos en los entornos que vayan más de la mano con la tecnología.

Los docentes en esta experiencia mostraron tanto en la encuesta como en el análisis del entorno virtual, una correcta y asertiva mediación pedagógica y espacios en donde se pueda desarrollar la criticidad, aunque más en una asignatura que en la otra.

\section{DISCUSIÓN}

El hecho de haber encontrado una diferencia entre la mediación pedagógica aplicada en las asignaturas puede indicar que hay más compromiso por parte de algunas tutoras, mientras que otras tienen una manera de trabajar que aún no satisface lo que la teoría establece sobre el personal docente a distancia. En un estudio realizado por Moulié y Díaz (2017), encontraron que uno de los factores que determina el desempeño del personal docente es su actitud. A partir de lo anterior, se recomienda motivar al personal a encontrar diferentes estrategias que permitan mayor participación por parte del estudiantado, las cuales se muestran al final de este apartado.

Con respecto a la capacitación, el hecho de que no todas las tutoras hayan llevado el curso "Diseño de cursos en línea", indica que no hay una uniformidad en el proceso de enseñanza-aprendizaje y esto puede incidir en la calidad de la asignatura que se imparte. En un estudio realizado por Hernández, Ariza y Cruz (2017), se demostró que las tecnologías de información facilitan la adquisición de conocimientos en el personal docente $y$, por ende, el estudiantado se ve beneficiado. Es por lo anterior que se recomienda uniformar esta situación para que se tengan las herramientas necesarias para realizar esta gestión de forma correcta.

Los resultados de esta experiencia coinciden con los de un estudio realizado por Peralta, Martínez, Arias e Intriago (2017), en el que se encontró que debe existir el acompañamiento académico con docentes especializados y con una comunicación fluida hacia el estudiantado. A pesar de que en esta experiencia se encontró una correcta y asertiva mediación pedagógica, hubo disparidad en los resultados obtenidos, lo cual se puede deber a que 


\section{CUADRO 2}

Resultados sobre la aplicación de la lista de chequeo en la asignatura Calidad Agroalimentaria por los dos expertos externos a la Cátedra.

\begin{tabular}{|c|c|}
\hline Característica deseada & Comentario de experto 1 \\
\hline $\begin{array}{l}\text { Mediación del personal docente } \\
\text { permite participación }\end{array}$ & $\begin{array}{l}\text { La mediación que realiza la tutora } \\
\text { está siempre enmarcada en el } \\
\text { respeto al estudiantado. }\end{array}$ \\
\hline $\begin{array}{l}\text { Uso correcto de la plataforma } \\
\text { virtual }\end{array}$ & $\begin{array}{l}\text { Hasta donde se puede observar, } \\
\text { hay un excelente dominio del uso } \\
\text { correcto de la plataforma virtual } \\
\text { Moodle. }\end{array}$ \\
\hline $\begin{array}{l}\text { Respuesta a tiempo de } \\
\text { preguntas }\end{array}$ & $\begin{array}{l}\text { En cada uno de los foros hay una } \\
\text { respuesta pronta y muy asertiva. }\end{array}$ \\
\hline
\end{tabular}

\section{Mediación del personal docente Revisando las unidades 2 y 3 hay un en los contenidos de los foros espacio académico donde existe una adecuada mediación.}

Espacios de socialización No hay evidencia de algún tipo

Comentario de experto 2

Tal como se ve en el entorno, las participaciones solicitadas son bastante cerradas, por ejemplo, se piden las fases de proceso y ejemplos, no hay mucho espacio para participación flexible.

Parece ser que se usa adecuadamente, pero sería preciso entrar conocer el programa del curso para saber si se hizo como se deseaba

En la mayoría de los casos, las respuestas son oportunas. Parece haber dos el personal docente porque se reciben respuestas de dos fuentes diferentes.

En el inicio de los foros no se siente esta mediación, y cuando se presenta es más como guía de la discusión que en los contenidos mismos. Eso sí, hay materiales con diferentes niveles de mediación.

Hay espacios preestablecidos para esto, pero no parecen ser muy usados, las dudas están en el mismo foro de de foro "Cafetería" donde el trabajo de las unidades. estudiantado puedan socializara con otros y puedan propiciar otros temas de conversación.

\begin{tabular}{|c|c|}
\hline Espacio de discusiones & $\begin{array}{l}\text { Sí hay espacios donde se da este } \\
\text { tipo de situaciones. }\end{array}$ \\
\hline Fomento de la criticidad & Sí se da \\
\hline $\begin{array}{l}\text { Incorporación de nuevas } \\
\text { tecnologías y recursos } \\
\text { innovadores }\end{array}$ & $\begin{array}{l}\text { En el entorno sólo hay evidencia de } \\
\text { archivos en formato de Word, y PDF. } \\
\text { Debe haber mayor equilibrio entre } \\
\text { documentos de PDF, audios, videos, } \\
\text { páginas web, videos cortos }\end{array}$ \\
\hline Material didáctico de calidad & $\begin{array}{l}\text { El material aportado es de buena } \\
\text { calidad. }\end{array}$ \\
\hline
\end{tabular}

Aunque no lo dice expresamente, es para esto que se usan dentro del curso visitado.

Lo más novedoso que vi es la solicitud de un infograma. archivos en formato de Word, y PDF. Debe haber mayor equilibrio entre documentos de PDF, audios, videos, calidad.

Por ejemplo, de APA pudo buscarse un mejor documento. Aunque es bueno que lean en inglés, estas normas tienen una versión para idioma español y tiene diferencias.

\begin{tabular}{lll}
$\begin{array}{l}\text { Organización del contenido } \\
\begin{array}{l}\text { Diseño de actividades } \\
\text { evaluativas }\end{array}\end{array}$ & $\begin{array}{l}\text { Es el correcto } \\
\text { Las actividades evaluativas que he } \\
\text { observado son tareas y foros, donde } \\
\text { hay un claro aprendizaje. }\end{array}$ & $\begin{array}{l}\text { Eso se diseña en las actividades iniciales, de aprendizaje y } \\
\text { de evaluación, solo en las últimas no es suficiente. }\end{array}$ \\
$\begin{array}{ll}\text { Diseño de instrumentos de } \\
\text { evaluación }\end{array}$ & $\begin{array}{l}\text { Hay evidencia en el entorno de una } \\
\text { evaluación donde el estudiantado } \\
\text { demuestra el conocimiento } \\
\text { adquirido durante el curso. }\end{array}$ & $\begin{array}{l}\text { Eso no se puede lograr ahí, es una estrategia de todo el } \\
\text { proceso de enseñanza y de aprendizaje. }\end{array}$ \\
\hline
\end{tabular}

la participación del estudiantado es mucho menor y no se llega a tener un nivel de discusión.

La falta la incorporación de nuevas tecnologías y recursos en los entornos que vayan más de la mano con la tecnología encontrados en esta experiencia, coinciden con el estudio realizado por González y Valenzuela (2017), en donde indican que la importancia de analizar sistemáticamente la acción e-tutorial proporciona información confiable, la cual propicia la identificación de oportunidades el favorecer la eficiencia del trabajo tutorial con la utilización de aplicaciones tecnológicas.

Es por lo anterior que se hace necesario incorporar más recursos que sean variados como videos, audios, links a artículos, teletutorías y reducir un poco la cantidad 


\section{CUADRO 3}

Resultados sobre la aplicación de la lista de chequeo en la asignatura Gerencia de Empresas Agropecuarias y Agroindustriales por un experto externo a la Cátedra

Característica evaluada

Mediación del personal docente permite participación

\begin{tabular}{|c|c|}
\hline Uso correcto de la plataforma virtual & $\begin{array}{l}\text { Durante el desarrollo del curso virtual hay una clara evidencia del dominio } \\
\text { de la plataforma. }\end{array}$ \\
\hline Respuesta a tiempo de preguntas & $\begin{array}{l}\text { En el foro de dudas, se observa que el personal docente responde a tiempo } \\
\text { las dudas de sus estudiantes. }\end{array}$ \\
\hline $\begin{array}{l}\text { Mediación del personal docente en los } \\
\text { contenidos de los foros }\end{array}$ & $\begin{array}{l}\text { Revisando cada uno de los temas que están disponibles }(1,2,3 \text { y } 4) \text { no se logra } \\
\text { demostrar una clara y adecuada mediación. }\end{array}$ \\
\hline Espacios de socialización & Solo hay un foro en todo el curso. \\
\hline Espacio de discusiones & No hay \\
\hline Fomento de la criticidad & No se logran visualizar espacios de discusión. \\
\hline $\begin{array}{l}\text { Incorporación de nuevas tecnologías y } \\
\text { recursos innovadores }\end{array}$ & $\begin{array}{l}\text { En el entorno solo hay evidencia de archivos en formato de Word, y PDF. Debe } \\
\text { haber mayor equilibrio entre documentos de PDF, audios, videos, páginas web, } \\
\text { videos cortos }\end{array}$ \\
\hline Material didáctico de calidad & El material aportado es de buena calidad. \\
\hline Organización del contenido & De acuerdo \\
\hline Diseño de actividades evaluativas & $\begin{array}{l}\text { Las tareas e informes están en el Tema } 3 \text {, y están bien diseñadas desde el punto } \\
\text { de vista técnico. }\end{array}$ \\
\hline Diseño de instrumentos de evaluación & $\begin{array}{l}\text { Hasta donde puedo ver en el entorno virtual, hay dos instrumentos visibles para } \\
\text { el estudiantado, donde hay una clara mediación. }\end{array}$ \\
\hline
\end{tabular}

Comentario de experto

Se observa en el foro de dudas un correcto y respetuoso trato con el estudiantado.

Durante el desarrollo del curso virtual hay una clara evidencia del dominio plataforma.

En el foro de dudas, se observa que el personal docente responde a tiempo Revisando cada uno de los temas que están disponibles $(1,2,3$ y 4$)$ no se logra

estudiantado, donde hay una clara mediación. de documentos en PDF que pueden ser largos y tediosos para el estudiantado, dando un equilibrio el cual es la clave para mejorar la calidad a la asignatura virtual (E. Chanto, comm pers, 13 de Julio, 2017).

Finalmente, el hecho de que dos de las tutoras hayan llevado capacitación en el tema de evaluar para aprender es muy beneficioso para la cátedra y el estudiantado, porque el evaluar para aprender, según Jiménez (2016), es un concepto de evaluación que surge de la conjunción de una serie de elementos clave extraídos de las concepciones de la evaluación como formativa y formadora.

Como recomendación, es importante fomentar en lel personal docente la construcción del conocimiento de manera colaborativa, lo cual se nota aún no se está realizando dado que no se trabaja con comunidades de aprendizaje y en una de las asignaturas no se encuentra una adecuada mediación. En un estudio realizado por Macías, Cañedo, Eudave, Páez y Carvajal (2017), encontraron que el personal docente considera que el trabajo colaborativo es un medio ideal para propiciar la reflexión y desarrollar nuevas competencias.
Se recomienda también fomentar espacios en la Cátedra, en donde se identifiquen las estrategias que se pueden utilizar para determinar las necesidades especiales que tienen el estudiantado de la cátedra, aun cuando la Oficina de Bienestar Estudiantil no lo mencione, porque aunque en la UNED de Costa Rica existe una oficina que provee la información del estudiantado con necesidades especiales, no todas las necesidades son de carácter académico, también pueden ser tecnológicas, geográficas o muy personales, que solamente conociendo a fondo a el estudiantado, se puede lograr superar barreras que ellos por desconocimiento o temor no logran superar y es parte de lo que la educación a distancia puede lograr. El Espacio Europeo de Educación Superior enfoque establece como referente considerar, en el marco de las competencias profesionales del profesor universitario, la necesidad de atender a la diversidad (Torelló \& Rueda, 2017).

Se concluye que esta experiencia ha dado una perspectiva muy importante sobre el personal docente de la UNED, la tarea de elaborar una tutoría, el desarrollo de las sesiones, son parte de las estrategias que respaldan una 
adecuada interacción entre los participantes que favorecen el trabajo colaborativo tutor-alumno.

Asimismo, se puede destacar como conclusión que la capacitación es una herramienta indispensable para potenciar las competencias tecnologías en los docentes, aunado a esto las capacitaciones deben de ir acompañadas de temas como la didáctica, pedagogía y mediación. De igual manera, el uso de las tecnologías permite generar espacios sincrónicos y asincrónicos, para fomentar la criticidad y el análisis del estudiantado de manera individual y colectiva.

\section{REFERENCIAS}

Cabero, J., \& Román, P. (2008) E-actividades. Un referente básico para la formación en Internet. Primera Reimpresión. Sevilla, España: Editorial MAD, S.L.

Calvo, X., \& Salas, N. (s.f.). Mediación pedagógica en entornos virtuales. En Umaña, A., Salas, I., \& Berrocal, V. (Ed)., Consideraciones para el diseño y oferta de asignaturas en línea (p. 34-42). San José, Costa Rica: EUNED.

Castillo, S., Torres, J., \& González, L. (2009) Tutoría en la enseñanza, la universidad y la empresa. Madrid: Ediciones UNED, Pearson Education.

García, L. (2014). Bases, mediaciones y futuro de la educación a distancia en la sociedad digital. Madrid, España: Editorial Síntesis.

González, E., \& Valenzuela, D. L. F. H. (2017). Una experiencia educativa, la e-tutoría en educación virtual con la aplicación del desarrollo tecnológico. Memorias del Encuentro Internacional de Educación a Distancia, 5(5), $1-23$.

Hernández, A., Ariza, G., \& Cruz, J. (2017). Educación a distancia: una modalidad factible para la capacitación docente del nivel medio superior. TEPEXI Boletín Científico de la Escuela Superior Tepeji del Rio, 4(7).

Jiménez, L. (2016). Marco teórico metodológico de la evaluación para aprender. Revista Calidad en la Educación Superior (CAES), 7(1), 100-126.

Macías, A., Cañedo, T., Eudave, D., Páez, D., \& Carvajal, M. (2017). Argumentación del personal docente de educación superior sobre su práctica y experiencia en el contexto del trabajo colaborativo. Investigación Cualitativa en Educación, 1, 791-799.

Moulié, M., \& Díaz, R. (2017). El formador en la modalidad educación a distancia: una mirada a su dimensión investigativa. REFCalE: Revista Electrónica Formación y Calidad Educativa, 5(1), 1-16.

Peralta, M., Martínez, J., Arias, E., \& Intriago, K. (2017). Sistema para las tutorías académicasen las universidades ecuatorianas. Caso Universidad Estatal de Milagro. INNOVA Research Journal, 2(6), 100-11.

Salas, I (s.f.). Modelo de Enseñanza y Formación en Línea. En Umaña, A., Salas, I., \& Berrocal, V. (Ed)., Consideraciones para el diseño y oferta de asignaturas en línea (p. 25-33). San José, Costa Rica: EUNED.

Torelló, O., \& Rueda, P. (2017). La atención a la diversidad en la educación superior: Una perspectiva desde las competencias docentes. Revista de Educación Inclusiva, 5(1), 159-174. 


\title{
APÉNDICE 1
}

\section{Encuesta sobre las competencias del personal docente}

\author{
Cátedra de Agroindustria \\ Universidad Estatal a Distancia
}

Estimado docente,

La siguiente es una encuesta que la Cátedra de Agroindustria está realizando para determinar las competencias que usted como tutor ha adquirido durante su experiencia en la UNED y cuáles aún no está aplicando según la teoría que respalda este tema.

\section{Consentimiento informado}

La información que usted provea en esta encuesta será tratada de manera confidencial y no tendrá injerencia alguna sobre su evaluación del desempeño o cualquier otro rubro que pudiera perjudicarle en su desempeño como tutor de la UNED.

\section{ENCUESTA}

¿Cómo planifica usted sus estrategias didácticas de manera que sean lo más apropiadas para que el estudiantado pueda realizarlas de manera autónoma y reflexiva?

( ) Identificando la manera que tiene cada estudiante de aprender para así determinar la mejor técnica que se puede utilizar para cada uno.

( ) Procurando que el estudiantado lleven a cabo procesos de interacción, reflexión y análisis de los contenidos.

( ) Monitoreando la participación de todos el estudiantado en las actividades, así como su avance.

¿Cuáles de las siguientes características utiliza usted para las asignaturas que imparte?:

( ) Crear ambientes en los cuales se potencie la formación de comunidades de aprendizaje.

( ) Estimular la construcción conjunta del conocimiento.

( ) Promover relaciones respetuosas entre estudiantes y estudiante-docente.

( ) Establecer áreas de consenso, reconociendo la diversidad de criterios y formas de interpretar el mundo.

( ) Realiza espacios de conocimiento mutuo fuera del área académica.

De las siguientes estratégicas pedagógicas, marque las que usted prefiere para impartir sus asignaturas

( ) Atender las dudas del estudiantado de forma cuidadosa, constante y a tiempo

( ) Crear ambientes de aprendizaje que estimulen el desarrollo de destrezas metacognitivas y de autorregulación en el estudiantado.

( ) Ser una guía en los procesos de aprendizaje tanto durante las actividades de plataforma como en las actividades de Scopia, tutorías presenciales, laboratorios o giras

( ) Desarrollar material o recursos que permitan atender necesidades especiales o reforzar conceptos.

( ) Potenciar el desarrollo del pensamiento reflexivo y crítico a partir de las actividades de aprendizaje que se propongan.

( ) Asegurar que las discusiones deriven en consensos y que cumpla con el objetivo propuesto.

¿Conoce usted el plan de estudios y el diseño curricular de las asignaturas correspondientes de la carrera?

( ) Sí

( ) No 
Utiliza usted material de apoyo a la hora de impartir sus tutorías presenciales y virtuales?. Si es así, de qué tipo?:

( ) Presentación de Power Point o Prezi

( ) Videos

( ) Artículos científicos

( ) Artículos de interés

Ha recibido el curso de Diseño y organización de cursos en línea en la UNED?

( ) $\mathrm{Si}$

( ) No

Qué otros cursos ha recibido relacionados con la innovación en la enseñanza de cursos en línea?

\author{
Gracias por su participación!
}




\title{
APÉNDICE 2
}

\section{Lista de chequeo para analizar las asignaturas en plataforma virtual}

\author{
Cátedra de Agroindustria \\ Universidad Estatal a Distancia
}

Estimado colega:

La siguiente es una lista de chequeo que la Cátedra de Agroindustria diseñó con el fin de analizar dos asignaturas del segundo cuatrimestre del año 2017, para las cuales había una plataforma virtual. Debe ser analizada a la luz de las competencias que un tutor debe tener en un entorno virtual.

\section{Consentimiento informado}

La información que usted provea en esta lista de chequeo será utilizada de manera confidencial.

Asignatura asignada:

\section{LISTA DE CHEQUEO}

\begin{tabular}{|c|c|c|}
\hline Característica & $\begin{array}{l}\text { Se encuentra presente } \\
\text { en el entorno virtual } \\
\text { de la asignatura? }\end{array}$ & Observaciones \\
\hline $\begin{array}{l}\text { La mediación del personal docente permite una } \\
\text { participación del estudiantado abierta y flexible }\end{array}$ & & \\
\hline $\begin{array}{l}\text { El personal docente demuestra un uso correcto de la } \\
\text { plataforma virtual en términos técnicos }\end{array}$ & & \\
\hline $\begin{array}{l}\text { El personal docente responde a tiempo las preguntas } \\
\text { del estudiantado }\end{array}$ & & \\
\hline $\begin{array}{l}\text { Se nota una mediación de los contenidos en los foros } \\
\text { de discusión por parte del profesor }\end{array}$ & & \\
\hline $\begin{array}{l}\text { El personal docente propicia espacios de socialización } \\
\text { entre estudiantes para fomentar la participación }\end{array}$ & & \\
\hline $\begin{array}{l}\text { El entorno virtual promueve espacios de discusión, } \\
\text { de orientación y de apoyo, más que la explicación de } \\
\text { contenidos }\end{array}$ & & \\
\hline $\begin{array}{l}\text { Las estrategias que utiliza el personal docente } \\
\text { fomentan la discusión, criticidad y son orientadas al } \\
\text { aprendizaje significativo }\end{array}$ & & \\
\hline $\begin{array}{l}\text { Se incorporaron nuevas tecnologías y recursos } \\
\text { innovadores en el entorno virtual }\end{array}$ & & \\
\hline $\begin{array}{l}\text { Se cuidó la calidad del material didáctico que se } \\
\text { colocó en la plataforma virtual }\end{array}$ & & \\
\hline $\begin{array}{l}\text { Los contenidos están organizados de manera } \\
\text { coherente y que permita una enseñanza didáctica de } \\
\text { la asignatura en la plataforma }\end{array}$ & & \\
\hline $\begin{array}{l}\text { Las actividades evaluativas están diseñadas de forma } \\
\text { que permiten un aprendizaje significativo? }\end{array}$ & & \\
\hline $\begin{array}{l}\text { Los instrumentos de evaluación están diseñados de } \\
\text { manera que se logre una mediación pedagógica }\end{array}$ & & \\
\hline
\end{tabular}

